\title{
Extraction of additional interfacial states of silicon nanowire field-effect transistors
}

\author{
Soshi Sato, ${ }^{1, a)}$ Wei Li, ${ }^{1}$ Kuniyuki Kakushima, ${ }^{2}$ Kenji Ohmori, ${ }^{3}$ Kenji Natori, ${ }^{1}$ \\ Keisaku Yamada, ${ }^{3}$ and Hiroshi Iwai ${ }^{1}$ \\ ${ }_{1}^{1}$ Frontier Research Center, Tokyo Institute of Technology, 4259-S2-20 Nagatsuta-cho, Midori-ku, \\ Yokohama 226-8502, Japan \\ ${ }^{2}$ Interdisciplinary Graduate School of Science and Engineering, Tokyo Institute of Technology, \\ 4259-S2-20 Nagatsuta-cho, Midori-ku, Yokohama 226-8502, Japan \\ ${ }^{3}$ Graduate School of Pure and Applied Sciences, University of Tsukuba, 1-1-1 Tennodai, Tsukuba, \\ Ibaraki 305-8573, Japan
}

(Received 20 March 2011; accepted 17 May 2011; published online 9 June 2011)

\begin{abstract}
Interfacial states of silicon nanowire field-effect transistors with rectangular-like cross-sections (wire height of $10 \mathrm{~nm}$ and widths of 9 and $18 \mathrm{~nm}$ ) have been evaluated from the transfer characteristics in the subthreshold region measured at cryogenic temperatures, where kinks in the drain current becomes prominent. It is found that the kinks can be well-explained assuming local interfacial states near the conduction band $\left(E_{\mathrm{c}}\right)$. The main extracted local states have been shown to exist at 10 and $31 \mathrm{meV}$ below $E_{\mathrm{c}}$ with the densities of $1.3 \times 10^{13} \mathrm{~cm}^{-2} / \mathrm{eV}$ and 5.4 $\times 10^{12} \mathrm{~cm}^{-2} / \mathrm{eV}$, respectively. By comparing two field-effect transistors with different wire widths, the former states can be assigned to the states located at the corner and the side surface of the wire, and the latter to the top and the bottom surfaces. (C) 2011 American Institute of Physics. [doi:10.1063/1.3598402]
\end{abstract}

Silicon nanowire (SiNW) metal-oxide-semiconductor field-effect transistor (MOSFET) is a promising candidate of future complementary MOS devices for further scaling. To improve the short channel effect immunity with SiNW FETs, the cross-sectional dimensions of SiNW channel should be designed to be small. ${ }^{1}$ As a result, curved surfaces near corners of rectangular-like SiNW cross-sections might be the dominant surface for the conduction as the cross-sectional dimensions decrease. ${ }^{2}$ In this case, curved surfaces with various surface orientations might be more prominent with smaller cross-sectional dimensions to modify the interfacial state density $\left(D_{\text {it }}\right)$ distribution energetically and physically different from that of planar-type FETs. ${ }^{3}$ As the interfacial states are related to reliability issues of gate oxide of MOSFET, ${ }^{4}$ it is important to characterize the interfacial states. In this letter, we report the interfacial state density distribution of SiNW $n$ FETs.

SiNW $n$ FETs with a semigate-around structure ${ }^{4}$ were fabricated on silicon-on-insulator (SOI) wafers. The detailed fabrication process is reported in Refs. 2 and 5. Crosssectional scanning transmission electron microscope images of the SiNW channels are shown in Figs. 1(a) and 1(b). SiNW $n$ FETs with rectangular-like cross-sections with channel widths $\left(w_{\mathrm{NW}}\right)$ of 9 and $18 \mathrm{~nm}$ were examined in this letter. The SiNW $n$ FETs had the same channel height $\left(h_{\mathrm{NW}}\right)$ of $10 \mathrm{~nm}$. Planar-type SOI $n$ FET with SOI layer thickness of $28 \mathrm{~nm}$ was also evaluated. Effective gate lengths $\left(L_{\mathrm{g}}\right)$ of the SiNW $n$ FETs with $w_{\mathrm{NW}}$ of 9 and $18 \mathrm{~nm}$, and planar-type SOI $n$ FET were $415 \mathrm{~nm}, 458 \mathrm{~nm}$, and $577 \mathrm{~nm}$, respectively, which were extracted by Chern's channel resistance method. ${ }^{6}$

\footnotetext{
${ }^{\text {a) }}$ Author to whom correspondence should be addressed. Electronic mail: sato@iwailab.ep.titech.ac.jp. Tel: +81-45-924-5847. FAX: +81-45-9245846.
}

Interfacial state density distribution was evaluated using transfer characteristics and subthreshold slope $(S S)$ of the SiNW $n$ FETs (Refs. 7 and 8) at a drain bias voltage of 50 $\mathrm{mV}$. The transfer characteristics were measured at temperatures from 290 down to $43 \mathrm{~K}$. SS is expressed as ${ }^{9}$

$$
S S=\ln 10 \times \frac{k T}{q}\left[1+\frac{C_{d}+C_{i t}\left(\phi_{s}\right)}{C_{o x}}\right],
$$

where $k$ is the Boltzmann constant, $T$ is the measurement temperature, $q$ is the elementary charge, $C_{\mathrm{ox}}$ is the oxide capacitance, $C_{\mathrm{d}}$ is the depletion capacitance, and $C_{\mathrm{it}}\left(\varphi_{\mathrm{s}}\right)$ is the interfacial state capacitance that is a function of the surface potential $\varphi_{\mathrm{s}} . C_{\mathrm{it}}\left(\varphi_{\mathrm{s}}\right)$ is a first order partial derivative of interface trap charge density $\left(Q_{\mathrm{it}}\right)$. The minimum $S S$ values

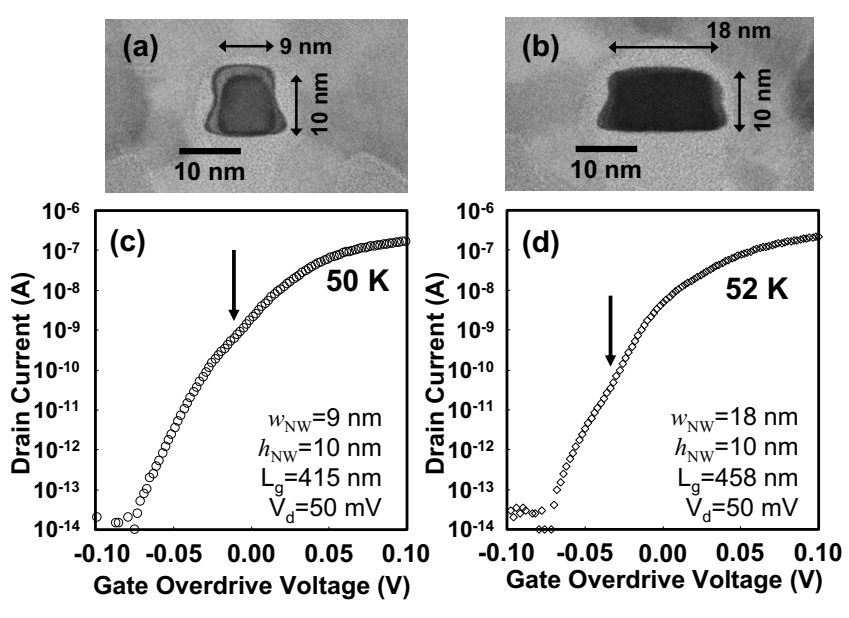

FIG. 1. Cross-sectional scanning transmission electron micrographs of the channels of SiNW FET with (a) $w_{\mathrm{NW}}$ of $9 \mathrm{~nm}$ and $h_{\mathrm{NW}}$ of $10 \mathrm{~nm}$ and (b) $w_{\mathrm{NW}}$ of $18 \mathrm{~nm}$ and $h_{\mathrm{NW}}$ of $10 \mathrm{~nm}$. Transfer characteristics of SiNW $n$ FETs with (c) $w_{\mathrm{NW}}$ of $9 \mathrm{~nm}$ and $h_{\mathrm{NW}}$ of $10 \mathrm{~nm}$ and (d) $w_{\mathrm{NW}}$ of $18 \mathrm{~nm}$ and $h_{\mathrm{NW}}$ of $10 \mathrm{~nm}$ are also shown. Kinks are indicated by arrows. 

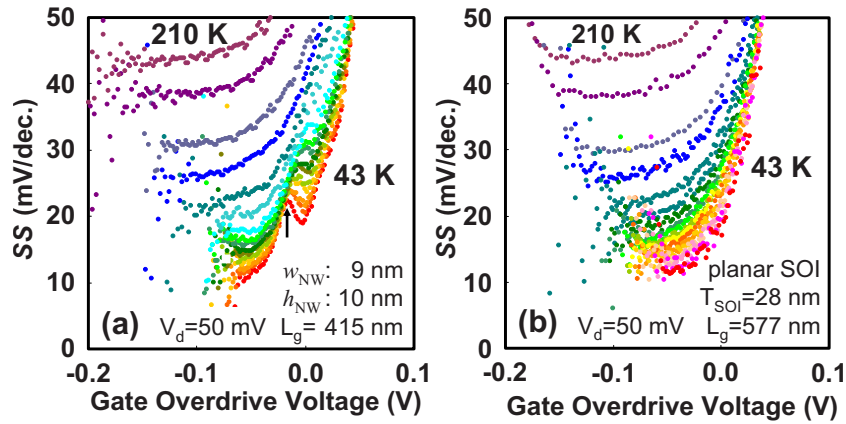

FIG. 2. (Color online) Subthreshold slopes of (a) SiNW $n$ FETs with $w_{\mathrm{NW}}$ of $9 \mathrm{~nm}$, and (b) planar-type SOI $n$ FET as a function of the gate overdrive voltages measured at temperatures from 43 to $210 \mathrm{~K}$.

at each temperature of SiNW $n$ FETs with $w_{\mathrm{NW}}$ of 9 and $18 \mathrm{~nm}$ showed linear relationship on measurement temperatures from 86 to $290 \mathrm{~K}$ in Eq. (1) with a slope of $2.0 \times 10^{-4} \mathrm{~V} / \mathrm{K}$ and $2.1 \times 10^{-4} \mathrm{~V} / \mathrm{K}$, which corresponds to constant $D_{\text {it }}$ of $2.9 \times 10^{11} \mathrm{~cm}^{-2} / \mathrm{eV}$ and 2.7 $\times 10^{11} \mathrm{~cm}^{-2} / \mathrm{eV}$, respectively. Moreover, at the measurement temperatures below $74 \mathrm{~K}$, kinks in transfer characteristics become predominant as shown in Figs. 1(c) and 1(d). Note that this feature was not observed for the planar-type SOI $n$ FET (not shown). SS of SiNW $n$ FET with $w_{\mathrm{NW}}$ of $9 \mathrm{~nm}$ and planar SOI $n$ FET were shown in Fig. 2. Based on Eq. (1), the kinks in the subthreshold slopes of the SiNW FET in Fig. 2(a) can be attributed to the increase in $C_{\text {it }}\left(\varphi_{\mathrm{s}}\right)$, which indicate localized interfacial states in the forbidden band gap of the SiNW channel. Therefore, a slight increase in the $V_{\mathrm{g}}$ may fill the local $D_{\mathrm{it}}$ with electrons $\left(Q_{\mathrm{it}}\right)$ to weaken the control of $\varphi_{\mathrm{s}}$, which causes the kink in the subthreshold slope. The kink appeared at higher gate overdrive voltage $\left(V_{\text {ov }}\right)$ as the measurement temperature increased. This is because larger increase in gate voltage is necessary to increase $\varphi_{\mathrm{s}}$ in strong inversion condition than in depletion and weak inversion conditions.

To extract the energy distribution of the interfacial states $D_{\text {it }}(E)$, subthreshold slopes were calculated assuming an arbitrary $D_{\text {it }}$ profile and compared with the obtained experimental data. A compact MOSFET model, Hiroshimauniversity STARC IGFET Model, ${ }^{10,11}$ was used for $S S$ calculation. Interfacial state density distribution was expressed as a sum of the Gaussian functions in Eq. (2);

$$
\begin{aligned}
& D_{i t}(E)=D_{i t, \text { peak }} \exp \left(\frac{E-E_{i t}}{2 \sigma^{2}}\right) \\
& Q_{i t}\left(\phi_{s}\right)=q \int D_{i t}(E) F\left(E, T, \phi_{s}\right) d E,
\end{aligned}
$$

where $E$ is the energy level in the silicon band gap, $F$ $\left(E, T, \varphi_{\mathrm{s}}\right)$ is the Fermi-Dirac distribution function, $D_{\text {it,peak }}$ is the maximum of the interfacial state density distribution, $\sigma$ is the variance of the Gaussian function, $E_{\mathrm{it}}$ is the center of localized interfacial state distribution. $C_{\mathrm{ox}}$ was extracted by split-CV method applied to a multichannel SiNW $n$ FETs, so that $1.64 \mu \mathrm{F} / \mathrm{cm}^{2}$ and $1.28 \mu \mathrm{F} / \mathrm{cm}^{2}$ were extracted for $9 \times 10 \mathrm{~nm}^{2}$ and $18 \times 10 \mathrm{~nm}^{2}$ SiNW $n$ FETs, respectively. The extracted $D_{\text {it }}(E)$ is shown in Fig. 3(a). One can observe two distinct peaks in the profile; a component $A$ with a peak interfacial state density of $1.3 \times 10^{13} \mathrm{~cm}^{-2} / \mathrm{eV}$ below 10
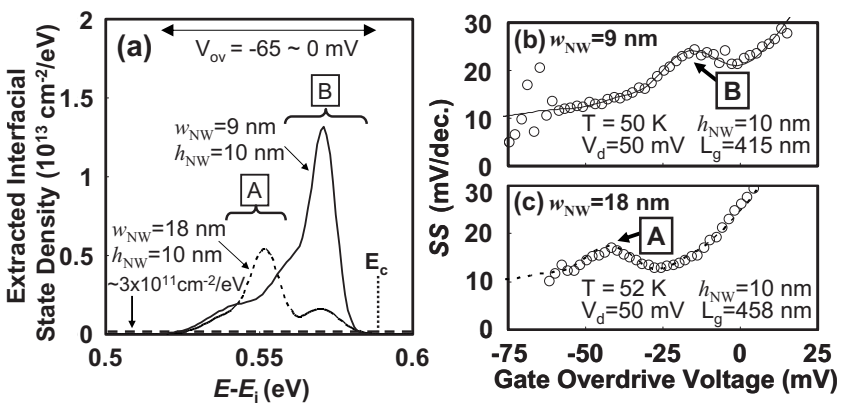

FIG. 3. (a) Interfacial state density of the SiNW $n$ FETs with $w_{\mathrm{NW}}$ of $9 \mathrm{~nm}$ (solid line) and $18 \mathrm{~nm}$ (dotted line) as a function of the energy levels in the band gap of the SiNW channels. Dashed line indicates constant interfacial state density in all energy levels. Subthreshold slopes of the SiNW $n$ FETs with $w_{\mathrm{NW}}$ of (b) $9 \mathrm{~nm}$ and (c) $18 \mathrm{~nm}$ obtained by experiments (open circle) and calculations (solid and dotted lines).

meV of $E_{\mathrm{c}}$, and a component $B$ with a peak interfacial state density of $5.4 \times 10^{12} \mathrm{~cm}^{-2} / \mathrm{eV}$ below $31 \mathrm{meV}$ of $E_{\mathrm{c}}$. The constant $D_{\text {it }}$ obtained from the minimum $S S$ measured at temperatures from 86 to $290 \mathrm{~K}$ are also shown in Fig. 3(a). These values are relatively small compared with the extracted $D_{\mathrm{it}}$. The calculated $S S$ explains well the measured $S S$ as shown in Figs. 3(b) and 3(c). A schematic band diagram for probing of the additional $D_{\mathrm{it}}(E)$ is shown in Fig. 4(a). While decreasing $w_{\mathrm{NW}}$ from 18 to $9 \mathrm{~nm}$, the component $B$ increased. On the other hand, the component $A$ was larger with larger channel width. These results suggest that the component $B$ is related to $D_{\text {it }}$ along the side surfaces including the upper and lower corners, and that the component $A$ is related to $D_{\text {it }}$ at the top surface as well as the bottom BOXnanowire interface as shown in Fig. 4(b), in which the coupling effect on the fully depleted SiNW $n$ FET was considered. ${ }^{12}$ Note that no kink was observed with planar SOI $n$ FET as shown in Fig. 2(b), indicating the specific $D_{\text {it }}$ distribution at the side surfaces or at the corners that have curved surface of the SiNW channel. There might be a concern that the surface potential fluctuation affected subthreshold slopes of MOSFET, ${ }^{13}$ which might introduce an error in the extracted $D_{\text {it }}$ distribution. Another concern might be that the components $A$ and $B$ affected mutually because of the coupling effects. ${ }^{12}$

It has been reported using the electron spin resonance that the trivalent silicon defect at silicon and silicon dioxide interface, which is called $P_{\mathrm{b}}$ center, is the origin of the interfacial states. ${ }^{14,15}$ Energy-resolved deep level transient spectroscopy is another evaluation method of interfacial states. ${ }^{16}$ Two kinds of $P_{\mathrm{b}}$ center, $P_{\mathrm{b} 0}$ and $P_{\mathrm{b} 1}$ have been proposed, and
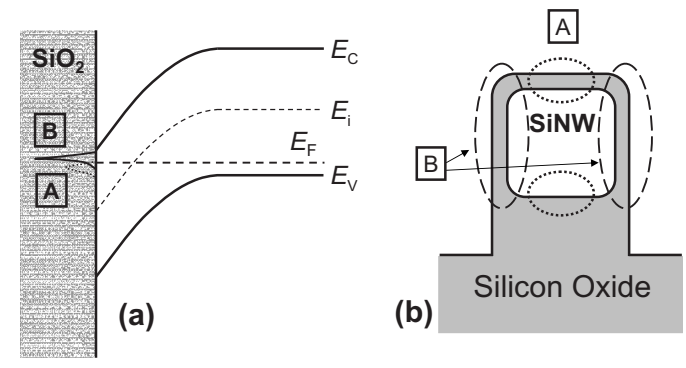

FIG. 4. (a) A schematic band diagram for probing of the localized interfacial states with components $A$ and $B$. (b) A schematic illustration for the relationship between the components of the additional interfacial states and the locations. 
the energy levels of $P_{\mathrm{b}}$ centers have been investigated by many authors. However, the peak energy levels of the interfacial state density distribution in Fig. 3(a) are not consistent with the peak energy levels of $P_{\mathrm{b} 0}$ and $P_{\mathrm{b} 1}$ on $(100),{ }^{16-20,23}$ $(110),{ }^{21,23}$ and (111)-oriented ${ }^{16,17,21-23}$ silicon/silicon dioxide interfaces. These facts indicate that the interfacial states of the SiNW $n$ FETs are physically different from those $P_{\mathrm{b} 0}$ and $P_{\mathrm{b} 1}$ on (100), (110), and (111)-oriented surfaces.

It has also been reported that stress was induced around the corners of the SiNW channel during gate thermal oxidation processes because of the volume expansion of silicon dioxide. $^{24,25}$ Ngai and White ${ }^{26}$ reported that the energy level of the interfacial defect changed as the distance between silicon atoms changed. One possible explanation to the interfacial states near the conduction band edge of the SiNW channel is that energy levels of the interfacial states $\left(P_{\mathrm{b}}\right.$ centers) split due to the distortion of the bond distances induced by the stress around corners during the thermal oxidation process.

Another possible reason might be the additional energy states due to the interstitial silicon atoms ${ }^{27}$ in the channel, as interstitial silicon atoms can be injected from the oxidation front into silicon beam structure during the thermal oxidation process. $^{28}$ The model suggests that interstitial silicon atoms near the oxide/channel interface injected by sacrificial oxidation and following gate oxidation processes, which generated energy states near conduction band edge that evaluated in this letter.

In summary, we reported the existence of the additional interfacial states of SiNW $n$ FETs with rectangular-like cross-sections (wire height of $10 \mathrm{~nm}$ and wire width of 9 and $18 \mathrm{~nm}$ ) from the transfer characteristics in the subthreshold region measured at cryogenic temperatures, where kinks in the drain current becomes prominent. It is found that the kinks can be well-explained assuming local interfacial states near the $E_{\mathrm{c}}$. The main extracted local states have been shown to exist at 10 and $31 \mathrm{meV}$ below the $E_{\mathrm{c}}$ with the densities of $1.3 \times 10^{13} \mathrm{~cm}^{-2} / \mathrm{eV}$ and $5.4 \times 10^{12} \mathrm{~cm}^{-2} / \mathrm{eV}$, respectively. By comparing two FETs with different wire widths, the former states can be assigned to the states located at the corner and the side surface of the wire, and the latter to the top and the bottom surfaces.

The authors thank all members of the ASKA II line and researchers in front-end program of R\&D Department 1 in Semiconductor Leading Edge Technologies, Tsukuba, for device fabrication and fruitful discussions. This work is supported by the program "Development of Nanoelectronic Device Technology" of the New Energy and Industrial Development Organization (NEDO).

${ }^{1}$ J.-P. Colinge, Solid-State Electron. 48, 897 (2004).

${ }^{2}$ S. Sato, K. Kakushima, P. Ahmet, K. Ohmori, K. Yamada, and H. Iwai, Microelectron. Reliab. 51, 879 (2011).

${ }^{3}$ M. Cassé, K. Tachi, S. Thiele, and T. Ernst, Appl. Phys. Lett. 96, 123506 (2010).

${ }^{4}$ D. J. DiMaria, E. Cartier, and D. Arnold, J. Appl. Phys. 73, 3367 (1993).

${ }^{5}$ S. Sato, H. Kamimura, H. Arai, K. Kakushima, P. Ahmet, K. Ohmori, K. Yamada, and H. Iwai, Solid-State Electron. 54, 925 (2010).

${ }^{6}$ J. G. J. Chern, P. Chang, R. F. Motta, and N. Godinho, IEEE Electron Device Lett. 1, 170 (1980).

${ }^{7}$ R. J. Van Overstraeten, G. J. Declerck, and P. A. Muls, IEEE Trans. Electron Devices 22, 282 (1975).

${ }^{8}$ J.-S. Lyu, K.-S. Nam, and C. Lee, Jpn. J. Appl. Phys., Part 1 32, 4393 (1993).

${ }^{9}$ J.-P. Colinge, Silicon-on-Insulator Technology: Materials to VLSI, 3rd ed. (Springer, New York, 2004).

${ }^{10}$ M. M.-Mattausch, H. J.-Mattausch, and T. Ezaki, The Physics and Modeling of MOSFETs: Surface-Potential Model HiSIM (World Scientific, Singapore, 2008).

${ }^{11}$ M. M.-Mattausch, U. Feldmann, A. Rahm, M. Bollu, and D. Savignac, IEEE Trans. Comput.-Aided Des. 15, 1 (1996).

${ }^{12}$ F. Dauge, J. Pretet, S. Cristoloveanu, A. Vandooren, L. Mathew, J. Jomaah, and B.-Y. Nguyen, Solid-State Electron. 48, 535 (2004).

${ }^{13}$ E. H. Nicollian and A. Goetzberger, Bell Syst. Tech. J. 46, 1055 (1967).

${ }^{14}$ Y. Nishi, Jpn. J. Appl. Phys. 10, 52 (1971).

${ }^{15}$ P. J. Caplan, E. H. Poindexter, B. E. Deal, and R. R. Razouk, J. Appl. Phys. 50, 5847 (1979).

${ }^{16}$ D. Vuillaume, D. Goguenheim, and G. Vincent, Appl. Phys. Lett. 57, 1206 (1990)

${ }^{17}$ E. H. Poindexter, G. J. Gerardi, M.-E. Rueckel, and P. J. Caplan, J. Appl. Phys. 56, 2844 (1984).

${ }^{18}$ G. J. Gerardi, E. H. Poindexter, and P. J. Caplan, Appl. Phys. Lett. 49, 348 (1986).

${ }^{19}$ B. J. O'Sullivan, P. K. Hurley, C. Leveugle, and J. H. Das, J. Appl. Phys. 89, 3811 (2001).

${ }^{20}$ J. P. Campbell and P. M. Lenahan, Appl. Phys. Lett. 80, 1945 (2002).

${ }^{21}$ P. K. Hurley, B. J. O'Sullivan, F. N. Cubaynes, P. A. Stolk, F. P. Widdershoven, and J. H. Das, J. Electrochem. Soc. 149, G194 (2002).

${ }^{22}$ P. K. Hurley, A. Stesmans, V. V. Afanas'ev, B. J. O'Sullivan, and E. O'Callaghan, J. Appl. Phys. 93, 3971 (2003).

${ }^{23}$ N. H. Thoan, K. Keunen, V. V. Afanas'ev, and A. Stesmans, J. Appl. Phys. 109, 013710 (2011)

${ }^{24}$ L. Sekaric, O. Gunawan, A. Majumdar, X.-H. Liu, D. Weinstein, and J.-W. Sleight, Appl. Phys. Lett. 95, 023113 (2009).

${ }^{25}$ A. Seike, T. Tange, I. Sano, Y. Sugiura, D. Kosemura, A. Ogura, and I. Ohdomari, Appl. Phys. Lett. 91, 062108 (2007).

${ }^{26}$ K. L. Ngai and C. T. White, J. Appl. Phys. 52, 320 (1981).

${ }^{27}$ G. M. Lopez and V. Fiorentini, Phys. Rev. B 69, 155206 (2004).

${ }^{28}$ A. M. Pyzyna, D. R. Clarke, and N. C. MacDonald, IEEE International Conference on Micro Electro Mechanical Systems, 2004, p. 189. 